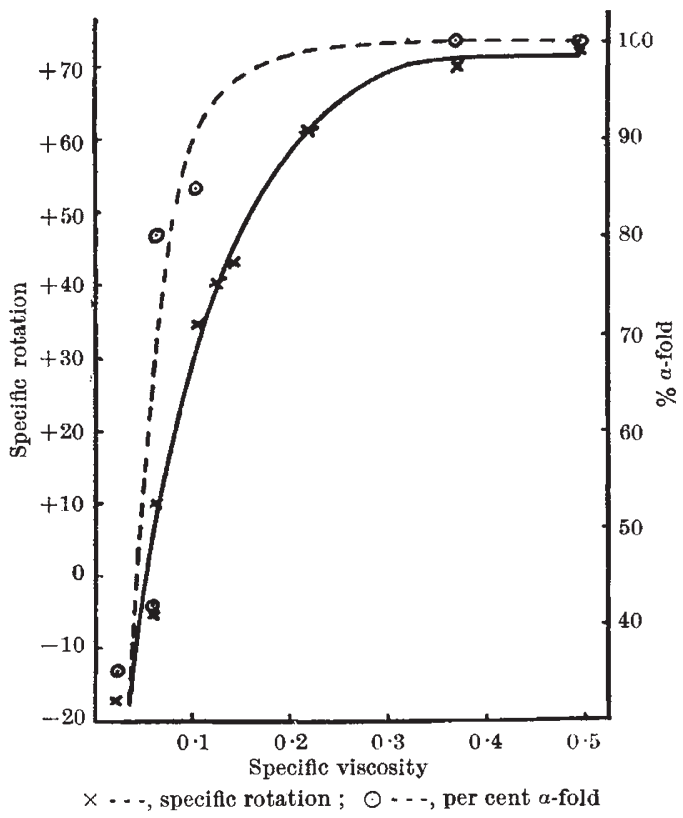

hot and cold evaporated films were found to be at $3,310 \mathrm{~cm} \cdot .^{-1}$ and $3,330 \mathrm{~cm} .^{-1}$ respectively.

These values suggest that the collagen fold is not present in the hot evaporated film; but unfortunately the data do not allow us to decide whether this film is in the $\alpha$ - or the $\beta$-configuration. The results, however, allow us to put forward a tentative picture of the gelling mechanism. Above $35^{\circ} \mathrm{C}$. gelatin would appear to exist in a form which does not allow hydrogen bonds to exist between the molecules. This condition would be satisfied either by the $\alpha$-fold or by a $\beta$-fold preferentially forming hydrogen bonds with the water. On concentrating such a hot solution there is little change in the optical rotation, which strongly suggests that it is in the $\alpha$-rather than in the $\beta$-configuration. Below $35^{\circ} \mathrm{C}$. the existence of the collagen fold becomes possible where lengths of parallel chain come into contact ; a three-dimensional network is built up consisting of lengths of single molecules joined together by small regions of parallel chains in the collagen configuration (the presence of more than 20 per cent proline and hydroxy-proline means, however, that there will be many residues which cannot be hydrogen-bonded in the $\alpha$-fold). Where the concentration and number of collagen folds cross-linked are sufficiently high, the network will permeate the whole solution, which will then set to a gel. On ageing, the organized regions in the collagen fold increase; this will continue until steric restrictions prevent the further growth of the organized regions. The ageing is accompanied by an increase in specific rotation and rigidity. On concentrating the gel there is a further large increase in the proportion of collagen fold, as indicated by the further marked increase in the optical rotation.

In attributing the origin of the gel formation to the formation of the collagen fold, we are assuming that any contribution from side-chain interaction will be of secondary importance. This is in accordance with the observation that the optical rotation ${ }^{5}$ as well as the melting point ${ }^{6}$ and rigidity ${ }^{7}$ of the gel are little dependent on $p H$.

If increasing quantities of certain salts (for example, lithium bromide, iodide or thiocyanate) are added to a cold gelatin solution, the specific rotation falls to a limiting value which is very near that for the hot solution, while at the same time gel formation is prevented $^{8}$. It would seem that high concentrations of such salts convert cold gelatin from the inter-chainhydrogen-bonded form to the intra-chain-hydrogenbonded $\alpha$-form. It is significant that lithium bromide, as Ambrose, Bamford, Elliott and Hanby ${ }^{\circ}$ have shown, converts silk from the inter-chain-hydrogenbonded $\beta$-form to the water-soluble $\alpha$-form.

Our thanks are due to Mr. E. J. Ambrose for having first directed our attention to the fact that a correlation between optical rotation and the chain configuration in polypeptides was to be expected from theories of optical rotatory power, to Dr. A. Elliott for obtaining the infra-red radiation data on gelatin, and to Mr. N. B. Abbott for that on poly. peptides. We also wish to thank Mr. W. E. Hanby for preparing the range of polypeptide specimens.

[March 7.

${ }^{1}$ Bamforl, C. H., Hanby, W. E., and Happey, F., Proc. Roy. Soc., A, 205,30 (195i).

${ }^{2}$ Ambrose, E. J., and Elliott, A., Proc. Roy. Soc., A, 205, 47 (1951).

${ }_{3}^{3}$ Bamford, C. H., Hanby, W. E., and Happey, F., Proc. Roy. Soc., A, 206, 407 (1951).

4 Ambrose, E. J., and Elliott, A., Proc. Roy. Soc., A, 208, 206 (1951).

"Carpenter, D. C., Dalhberg, A. C., an'́d Hening, J. C., Indust. Eng. Chem., 20, 397 (1928).

' Pleass, W. B., Proc. Roy. Soc., A, 126, 406 (1930).

' Gerngross, A., Kolloid-Z., 40, 279 (1926).

${ }^{8}$ Carpenter, D. C., and Lovelace, F. F., J. Amer. Chem. Soc., 57, 2337 (1935).

- Ambrose, E. J., Bamford, C. H., Elliott, A., and Hanby, W. E., Nature, 167, 264 (1951).

\section{A HUNDRED YEARS OF ORGANIC CHEMISTRY (I85I-195I)*}

7 HE century 1851-1951 has been an eventful one for organic chemistry. Indeed, it has seen the science grow from a rather confusing and empirical body of facts into one of the most systematic and fascinating of the sciences, and one on the progress of which many of the amenities of our modern civilization depend.

1851 might almost be regarded as a turning point in organic chemistry, for major developments destined to shape the whole course of the science were at hand. It will be recalled that early in the nineteenth century Berzelius had defined organic chemistry as the chemistry of substances produced by living matter, in contradistinction to the inorganic substances of mineral origin. There were certain striking differences between the two types, and in Berzelius's mind, as in those of his contemporaries, these were associated with the vital force concept. Wöhler's laboratory synthesis of urea in 1828 is generally believed to have sounded the death knell of the vital force theory ; but, in fact, it survived for long after that and it still rears its head in the famous text-book of Gmelin (1854). In the years that followed Wöhler's discovery, however, Liebig and others had shown that the organic compounds all contained carbon and that carbon compounds with similar properties could be obtained from non-living sources, for example, coal tar. So it gradually came to be recognized that organic chemistry was, in effect, synonymous with the chemistry of the carbon compounds, and attention was directed to the understanding of carbon chemistry, since it was clear that

* Substance of a paper read by Prof. A. R. Todd, F.R.S., before 
until such an understanding was obtained little progress was to be expected in studying the chemistry of living matter.

By 1851 the efforts of many chemists had produced a substantial body of factual knowledge ; but despite type theories and radical theories, there was no really satisfactory theory of valency which could correlate the facts and establish the interrelationships of organic compounds. In 1852 E. Frankland made the first clear statement of the idea of valency or fixed combining power of an element. This was a great step forward, and it was followed in 1858 by the work of Kekulé and Couper, who independently put forward a theory of molecular structure based on the quadrivalency of carbon and the capacity of carbon atoms for joining together in chains; they showed how molecular constitution could be represented diagrammatically in graphic formulæ and could predict the existence of isomers. That more isomers did, in fact, exist than were allowed for by this theory was, however, clear from the earlier work of Pasteur, who had shown that two optically active tartaric acids exist. This was clarified in 1874 by van 't Hoff and Le Bel, who added to the KekuléCouper ideas the concept of the tetrahedral carbon atom and thereby introduced the idea of space- or stereo-isomerism. With this the whole subject was placed on a firm basis, and on these theories virtually the whole of the modern science arose. Only in comparatively recent years have alterations in basic ideas of molecular structure come from physics through knowledge of the electronic structures of atoms, but even these new ideas have not altered the essential applicability of theory laid down between 1850 and 1874 .

Armed with this understanding of structure, the study of the carbon compounds flourished exceed. ingly, and by the early twentieth century a vast number of such compounds were known and methods of synthesis and analysis had been developed in great variety. During this period, too, began the rise of the great organic chemical industry-producing dyestuffs, starting from the initial aniline dye of Perkin, explosives, etc. This rise of an industry notable for its eloseness to the advancing front of a science has continued, until to-day it produces not only dyes and explosives, but also plastics, detergents, photographic materials, drugs, fabrics and a multitude of other things which we accept without question as part of everyday life - there are indeed few of the material comforts of life which do not owe something to organic chemical industry.

The twentieth century has seen, however, a remarkable development in our knowledge of chemistry in relation to living matter. During the latter half of the nineteenth century organic chemists paid scant attention to this side of their subject, and even biochemists-those concerned primarily with the nature of the processes going on in living matter-were relatively few. It is only in this century that great strides in biochemistry have been made.

From the organic chemist's point of view, this was inevitable. The materials found in living matter were too complex for him to study with success until he had mastered the groundwork and developed methods for studying carbon compounds in general. This mastery came from the work of the late nineteenth century, and as the twentieth begins we can see the beginnings of a move towards the goal of the early organic chemists in the work of Fischer,
Willstätter and others on the proteins, purines, sugars, natural colouring matters and enzymes. This movement has grown continuously, receiving enormous impetus through the development of microtechniques enabling work to be done with tiny quantities of material ; and in the past twenty-five years it has given most of the spectacular achievement of organic chemistry in the structural elucidation and synthesis of hormones, vitamins, alkaloids, natural colouring matters, etc., and indirectly of the synthetic drugs. Parallel with it have come the developments in the understanding of the finer details of molecular structure and reactivity associated with the names of Lapworth, Robinson, Pauling and Ingold. The welding together of the results and ideas emerging from these two fields of endeavour promise still more striking triumphs for organic chemistry in a future which should be as fascinating as its past.

\section{VERTICAL MIGRATION OF PLANKTON ANIMALS*}

$M$ ANY of the small plankton animals in the sea which are important as the food of fish such as herring, sprat and mackerel swim upwards towards the surface in the evening and down again to deeper levels after dawn. It is of interest from a purely biological point of view to find out what are the factors which govern these movements, and may also be useful in reaching a better understanding of the shoaling of the herring. The plankton consists of small animals of many different kinds, small jellyfish, worms and molluses, hosts of small crustaceans and many others; they nearly all show this nightly vertical migration upwards. Since it has been developed in so many different groups of animals and must use up so much energy every day-some of them climbing more than a hundred feet-it must clearly be of profound significance in their lives. We do not yet understand its meaning and are still only in the stages of studying the actual movements of the animals in relation to different conditions of light, temperature, pressure, etc.

Hitherto it has not been possible to see animals making extensive vertical migrations in the laboratory or to experiment with them while doing so. Now apparatus has been devised which allows this to be done. A curved transparent tube of 'Perspex', of 2 in. by $1 \frac{1}{2}$ in. cross-section, is made to form a complete circle of 4 -ft. diameter and is then mounted as a wheel; a small opening, which may be sealed up, allows it to be filled with water and the experimental animal added. The wheel is now turned so that the animal is half-way up one side; it can now swim up or down according to the varying conditions of light, etc., which may be controlled; but as it does so the wheel is turned so that it is actually kept stationary in relation to the observer. It is seen that the animal is swimming in an endless tube; it can swim up and up, or down and down for hundreds of feet, and the wheel, which is turned all the time, automatically records its speed of swimming on a recording drum. Inside the tube are small doors, some with weights and some with floats, which automatically open and close as the wheel goes round; they are always wide

* Substance of a paper read by Prof. A. C. Hardy, F.R.S., and R. Bainbridge before Section D (Zoology) of the British Association on August 10. 